\title{
Gli family zinc finger 1 is associated with endothelin receptor type $B$ in Hirschsprung disease
}

\author{
WEIZHEN LIU ${ }^{1}$, JUAN PAN $^{2}$, JINBO GAO $^{1}$, XIAOMING SHUAI $^{1}$, \\ SHAOTAO TANG $^{3}$, GUOBIN WANG $^{1}$, KAIXIONG TAO $^{1}$ and CHUANQING WU ${ }^{1}$ \\ Departments of ${ }^{1}$ Gastrointestinal Surgery, ${ }^{2}$ Pathology and ${ }^{3}$ Pediatric Surgery, Union Hospital, \\ Tongji Medical College, Huazhong University of Science and Technology, Wuhan, Hubei 430022, P.R. China
}

Received July 26, 2017; Accepted November 23, 2017

DOI: $10.3892 / \mathrm{mmr} .2018 .8612$

\begin{abstract}
Hirschsprung disease (HSCR) is a newborn colorectal disease characterized by an absence of ganglia in the distal gut. Hedgehog $(\mathrm{Hh})$ and endothelin signaling serve important roles in gastrointestinal tract formation. Alterations in the signaling pathways disrupt the development of enteric neural crest cells (ENCCs). It is not known whether there is any coordination between these pathways in the pathogenesis of HSCR. In the present study, tissue samples from 35 patients with HSCR, including stenotic aganglionosis gut and normal ganglionic gut, were obtained. The expression of Gli family zinc finger 1 (Gli1) and endothelin receptor type B (EDNRB) was determined using reverse transcription-quantitative polymerase chain reaction, immunohistochemistry and western blotting. In addition, the SK-N-SH cell line was used to investigate the association between Hh signaling and the expression of EDNRB. The results revealed aberrant expression of Gli1 in the aganglionic segments, as well as decreased expression of Glil in tissues from 7 patients with HSCR exhibited, whereas tissues from 9 patients with HSCR exhibited increased Gli1 expression compared with the expression in the normal tissues. There was a negative association between EDNRB expression and Gli1 expression in the same sample. Knockdown of Gli1 by small interfering RNA and inhibition of Hh signaling by Vismodegib in SK-N-SH cells increased EDNRB expression. By contrast, upregulation of Glil expression by plasmids and activation of $\mathrm{Hh}$ signaling by Purmorphamine decreased EDNRB expression. Furthermore, premature enteric ganglia were observed in 4 patients with HSCR with decreased Gli1 expression. Thus, the results of the present study suggest that altered Gli1 expression negatively regulates EDNRB
\end{abstract}

Correspondence to: $\mathrm{Dr}$ Chuanqing $\mathrm{Wu}$, Department of Gastrointestinal Surgery, Union Hospital, Tongji Medical College, Huazhong University of Science and Technology, 1277 Jiefang Avenue, Wuhan, Hubei 430022, P.R. China

E-mail: wucq2014@hust.edu.cn

Key words: Hirschsprung disease, hedgehog signaling pathway, endothelin receptor type B, enteric neural crest cells expression in patients with HSCR. The increased expression of EDNRB induced by decreased Gli1 expression may represent a novel mechanism in HSCR.

\section{Introduction}

Hirschsprung disease (HSCR) is a congenital digestive disease with an incidence of $\sim 1 / 5,000$ at birth (1). Distal stenotic gut and proximal dilated gut are common symptoms of the disease $(2,3)$, which are caused by abnormal development of the enteric nervous system (ENS) during embryogenesis (4). HSCR may be categorized into different phenotypes according to the aganglionic gut length, including short segment HSCR, long segment HSCR and total colonic aganglionosis (5). A number of definitive genes contribute to the migration and differentiation of enteric neural crest cells, including ret proto-oncogene (RET), glial cell line derived neurotrophic factor (GDNF), endothelin 3 (EDN3)/endothelin receptor type B (EDNRB), Hedgehog (Hh) and sex determining region Y-box 10 (6-8). However, the complex inheritance patterns of HSCR remain unclear. Mutations in these susceptibility genes account for $<50 \%$ of familial HSCR and a small proportion of sporadic cases (9). Hence, relevant signaling pathway cross-talk is likely to contribute to the pathogenesis of HSCR.

The Hh signaling pathway is a highly conserved pathway that serves a role in the development of organs in a range of organisms from drosophila to humans; its malfunction is associated with various diseases, including developmental diseases and carcinomas (10). The transcriptional factors of the Gli family in mammals, including GLI family zinc finger 1 (Gli1), Gli2, and Gli3 serve important roles in the Hh signaling pathway. Gli1 and Gli2 are primary activators, which are generally activated by the Hh-Patched-Smoothened pathway, whereas Gli3 predominantly acts as a transcriptional repressor $(11,12)$. Hh is a key molecule serving a role in enteric neural crest cell (ENCC) migration and differentiation and the pathogenesis of HSCR (13). During gut development, inhibition of the extracellular matrix protein sonic hedgehog (Shh), which is derived from gut epithelium, leads to intestinal hyperganglionosis (14). In Shh ${ }^{-/-}$and Indian hedgehog ${ }^{-/}$mice, there are multiple gastrointestinal defects, including malrotation of the gut as well as smooth muscle and distinct ENS defects (15). It has been demonstrated that Shh also inhibits ENCC migration 
and differentiation by eliminating the role of GDNF (16). Similarly, distal aganglionic gut and proximal hypoganglionic gut were observed with the addition of Shh in organ culture. Furthermore, the results of a sequencing and functional study have demonstrated that a novel gain-of-function mutation in Gli was identified in HSCR and aberrant Gli activity delayed gut colonization of ENCCs, which account for the HSCR phenotype (17). Inconsistencies in the results indicate that the role served by the Hh signaling pathway in HSCR is complex.

EDNRB belongs to a super-family of G-protein coupled receptors and is activated by the EDN3 ligand (18). The EDN3/EDNRB signaling pathway is essential for normal ENCC migration and differentiation and is generally accepted as a major susceptibility gene for HSCR $(19,20)$. Mutations in the EDNRB gene have been linked to HSCR in mice and humans (21). In EDNRB knockout mice (Ednr $\left.b^{\text {flex3/flex3 }}\right)$, ENCC migration is delayed; the EDNRB specific-antagonist BQ-788 reduced wild-type ENCC mobility and disrupted wavefront migration (22). A study involving a novel HSCR mouse model Ednrb $^{\text {mlyzcm }}$, which is caused by the L286P mutation in the TM $\mathrm{V}$ domain of the EDNRB, indicated an association between EDNRB and HSCR (23). Furthermore, active EDNRB induces HSCR in humans. EDNRB expression is upregulated in the aganglionosis segment of patients with HSCR and has a significantly lower ratio of methylation in HSCR (24).

Genetic alterations associated with mutations and expression levels, are the key components of Hh signaling and EDN3/EDNRB affect ENCC migration and differentiation, representing a primary etiology for HSCR (7). Theoretically, the cumulative effects of several disordered pathways are more likely to lead to disease. However, the functions of these signaling pathways in the cooperative modification of HSCR phenotypes and the mutual regulatory mechanism remain unknown.

The aim of the present study was to further demonstrate the mechanism by which the Hh signaling pathway negatively regulates EDNRB expression in HSCR. Downregulation of Gli1 followed by increased expression of EDNRB hinders the differentiation of ENCCs. By contrast, upregulation of Gli1 accompanied with decreased expression of ENDRB inhibits ENCCs proliferation and migration as reported in the present study. Furthermore, the results of the present study suggest that EDNRB is downstream of Gli1 and regulates ENCC development in the developing intestine and that an appropriate expression level is required for intestinal development. To the best of our knowledge, the present study is the first to demonstrate the mutual effect of Hh/EDNRB in HSCR development. The results herein may aid in identifying a novel disease mechanism for HSCR.

\section{Materials and methods}

Tissue sample preparation. A total of 35 samples from patients with HSCR, including the distal stenotic gut and the proximal normal gut were collected between April 2015 and April 2016 at the Union Hospital (Wuhan, China). Confirmed HSCR patients were enrolled into the present study and HSCR patients with other diseases were excluded. All patients (age, 9.57 \pm 8.32 months; 27 males, 8 females) with HSCR were diagnosed by barium enema prior to surgery and postoperative pathological diagnosis. Each tissue sample was divided into several sections. One section from each specimen was immediately fixed in $4 \%$ paraformaldehyde at room temperature for $24 \mathrm{~h}$ and embedded with paraffin. The other sections were frozen at $-80^{\circ} \mathrm{C}$ for molecular analysis. The current study was approved by the Ethics Committees of Union Hospital of Huazhong University of Science and Technology and was performed according to the Declaration of Helsinki. Specimens were collected after written informed consent was obtained from patient guardians.

Reagents and antibodies. Dimethyl sulfoxide (DMSO) was obtained from Sigma-Aldrich (Merck KGaA, Darmstadt, Germany). Vismodegib (cat no. s1082) and Purmorphamine (cat no. s3042) was obtained from Selleck Chemicals (Houston, TX, USA). Lipofectamine ${ }^{\circledR} 3000$ was purchased from Thermo Fisher Scientific, Inc. (Waltham, MA, USA). Antibody against Gli1 (cat no. sc-20687) was purchased from Santa Cruz Biotechnology, Inc. (Dallas, TX, USA). Antibody against EDNRB (cat no. ab117529) was purchased from Abcam (Cambridge, MA, USA). Antibody against GAPDH (cat no. 10494-1-AP) was purchased from Wuhan Sanying Biotechnology (Wuhan, China). The secondary goat anti-rabbit antibody (cat no. 7074S) was purchased from Cell Signaling Technology (Danvers, MA, USA). The H\&E staining kit (cat no. AR1180), immunohistochemical SABC reagent kit (cat no. SA1020), DAB (cat no. AR1022) and normal goat serum (cat no. AR0009) were purchased from Wuhan Boster Biological Technology, Ltd. (Wuhan, China).

Cell culture and treatment. The human neuroblastoma cell line SK-N-SH (Cell Bank of the Chinese Academy of Sciences, Shanghai, China) was cultured in Dulbecco's modified Eagle's medium (Thermo Fisher Scientific, Inc.) supplemented with $10 \%$ fetal bovine serum (Gibco; Thermo Fisher Scientific, Inc.), $100 \mathrm{U} / \mathrm{ml}$ penicillin and $100 \mu \mathrm{g} / \mathrm{ml}$ streptomycin (Thermo Fisher Scientific, Inc.). Cells were cultured in a humidified environment containing $5 \% \mathrm{CO}_{2}$ at $37^{\circ} \mathrm{C}$. The culture medium was replaced every day.

A total of $5 \mathrm{mg}$ Vismodegib was dissolved in $594.4 \mu \mathrm{l}$ DMSO and $5 \mathrm{mg}$ Purmorphamine was dissolved in $960.4 \mu \mathrm{l}$ DMSO. Cells were cultured in 6-well plates at a density of $2 \times 10^{5}$ cells/well for $12 \mathrm{~h}$. Cells were subsequently treated with Vismodegib (5, 10 and $20 \mu \mathrm{M})$ or Purmorphamine $(2.5,5$ and $10 \mu \mathrm{M})$ for $48 \mathrm{~h}(25)$. DMSO $(\leq 0.4 \%)$ was used as a blank control.

Small interfering (si)RNA and plasmid transfection. A total of $20 \times 10^{4}$ SK-N-SH cells were transfected with $100 \mathrm{nM}$ siRNA using Lipofectamine ${ }^{\circledast} 3000$ according to the manufacturer's protocol. After $48 \mathrm{~h}$ post-transfection, cells were harvested by trypsinization and centrifugation $(12,000 \mathrm{x} \mathrm{g}, 5 \mathrm{~min})$ at $4^{\circ} \mathrm{C}$. Gli1 siRNA and negative control were purchased from Guangzhou RiboBio Co., Ltd., (Guangzhou, China). The following target sequences were used: Gli1, 5'-GACACTGTT TCCTGATAAT-3'; negative control, 5'-UUCUCCGAACGU GUCACGU-3'. The Gli1 expression plasmid was purchased from Shanghai GenePharma Co., Ltd. (Shanghai, China; accession no. NM_005269). Cells were transfected with $1 \mu \mathrm{g} / \mathrm{ml}$ plasmid and treated with puromycin to select the stably transfection cells expressing puromycin-resistant genes. 
Table I. Primer sequences.

\begin{tabular}{lll}
\hline Gene & \multicolumn{1}{c}{ Forward } & Reverse \\
\hline GAPDH & 5'-AAGAAGGTGGTGAAGCAGG-3' & 5'-GTCAAAGGTGGAGGAGTGG-3' \\
Gli1 & 5'-TTGTCCCACACCGGTACCAC-3' & 5'-CCTCGCTCCATAAGGCTCAG-3' \\
EDNRB & 5'-CATCGTCATTGACATCCCTAT-3' & 5'-TTCTACTCCAAGAAGCAACAGC-3'
\end{tabular}

Gli1, Gli1 family zinc finger 1; EDNRB, endothelin receptor type B.

$H \& E$ staining and immunohistochemistry. Tissue sections (5 $\mu \mathrm{m}$ thick) were cut from paraffin blocks, dewaxed with xylene and rehydrated in a graded series of alcohol. According to the H\&E staining kit protocols, the procedures included 5 min hematoxylin staining, 5 min washing with water, $30 \mathrm{sec}$ soaking in $95 \%$ alcohol, and 2 min eosin staining. For immunohistochemistry, antigen retrieval at $95^{\circ} \mathrm{C}$, using citrate buffer and blocking with $10 \%$ normal goat serum for $1 \mathrm{~h}$ at room temperature was performed. Sections were incubated with primary antibodies (anti-Gli1, 1:50; anti-EDNRB, 1:200) overnight at $4^{\circ} \mathrm{C}$. Following three washes with PBS for 5 min each, sections were incubated with the secondary antibody from the SABC reagent kit for $30 \mathrm{~min}$ at $37^{\circ} \mathrm{C}$. Sections were treated with SABC solution and incubated with DAB according to the SABC reagent kit instructions. Primary antibody diluent was used as a negative control. The images were captured under a microscope (magnification, x100; Nikon ECLIPSE 80i, Nikon Corporation, Tokyo, Japan).

RNA isolation and reverse transcription-quantitative polymerase chain reaction ( $R T-q P C R)$. Total RNA was extracted from cells and tissue samples using TRIzol reagent (Thermo Fisher Scientific, Inc.) and reverse transcribed $\left(37^{\circ} \mathrm{C}\right.$ for $15 \mathrm{~min}$, followed by $85^{\circ} \mathrm{C}$ for $5 \mathrm{sec}$ ) into cDNA using PrimeScript RT Master mix (cat no. RR036A; Takara Biotechnology Co., Ltd., Dalian, China). SYBR ${ }^{\circledR}$ Premix Ex TaqTM (cat no. RR420A; Takara Biotechnology Co., Ltd.) was used to detect the mRNA level on the StepOnePlus ${ }^{\mathrm{TM}}$ Real-Time PCR system (Applied Biosystems; Thermo Fisher Scientific, Inc.). Target sequences were amplified at $95^{\circ} \mathrm{C}$ for $5 \mathrm{sec}$, followed by 40 cycles of $95^{\circ} \mathrm{C}$ for $5 \mathrm{sec}$ and $60^{\circ} \mathrm{C}$ for $30 \mathrm{sec}$. Each experiment was repeated in triplicate. GAPDH was used as an internal control. The expression levels of Gli1 and EDNRB were calculated using the $2^{-\Delta \Delta \mathrm{Cq}}$ method (26). The primer sequences are presented in Table I.

Western blot analysis. Following treatment with Vismodegib, Purmorphamine or DMSO for $48 \mathrm{~h}$, cells were lysed in radioimmunoprecipitation assay buffer (Sigma-Aldrich; Merck KGaA) supplemented with $1 \mathrm{mM}$ proteinase inhibitor (Beyotime Institute of Biotechnology, Haimen, China). Protein concentrations were determined using a bicinchoninic acid protein assay kit (Beyotime Institute of Biotechnology). A total of $40 \mu \mathrm{g} /$ lane protein was separated by $8 \%$ SDS-PAGE and transferred to polyvinylidene fluoride membranes (EMD Millipore, Billerica, MA, USA). Following blocking for $1 \mathrm{~h}$ with $5 \%$ skimmed milk at room temperature, membranes were incubated with primary antibodies (anti-Gli1, 1:200; anti-EDNRB, 1:1,000; anti-GAPDH, 1:5,000) overnight at $4^{\circ} \mathrm{C}$.
Table II. Patient demographic features.

\begin{tabular}{lcc}
\hline Features & Number & Ratio \\
\hline Age (months, mean \pm & $9.57 \pm 8.32$ & \\
standard deviation) & & \\
Sex & & $3.375: 1$ \\
Male & 27 & \\
Female & 8 & \\
\hline
\end{tabular}

Membranes were then washed with TBST and incubated with the secondary goat anti-rabbit antibody $(1: 4,000)$ for $1 \mathrm{~h}$ at room temperature. An enhanced chemiluminescence kit (Beyotime Institute of Biotechnology) was used to verify the bands. The ChemiDoc ${ }^{\mathrm{TM}}$ XRS+ System with Image $\mathrm{Lab}^{\mathrm{TM}}$ Software v6.0 (cat no. 170-8265, Bio-Rad Laboratories, Inc. Hercules, California, USA) was used to capture the images. All experiments were performed in triplicate.

Statistical analysis. Data are presented as the mean \pm standard deviation. Statistical analysis was performed using SPSS v.20.0 (IBM Corp., Armonk, NY, USA) and presented using GraphPad Prism 5.0 software (GraphPad Software, Inc., La Jolla, CA, USA). Comparisons between two groups were determined by paired t-tests. One way analysis of variance following by a Tukey's post hoc test was used to analyze differences among $>2$ groups. The median Gli1 and EDNRB expression between matched normal and stenotic gut samples was analyzed using a Wilcoxon signed-rank test. $\mathrm{P}<0.05$ was considered to indicate a statistically significant difference.

\section{Results}

Clinical data analysis. A total of 35 tissue specimens from patients with HSCR who were diagnosed by barium enema and anorectal manometry evaluation prior to surgery, as well as post-operative pathological examination, were included in the present study. The proximal normal tissues were used as controls. Clinical information, including age and sex, is presented in Table II. The mean age was 9.57 \pm 8.32 months. The male: Female ratio among patients with HSCR was 27:8, which is consistent with the typical sex ratio of this disease (3.5:1 4:1) (27).

Expression of Glil and EDNRB in HSCR. The potential significance of the Hh signaling pathway in the development 
A

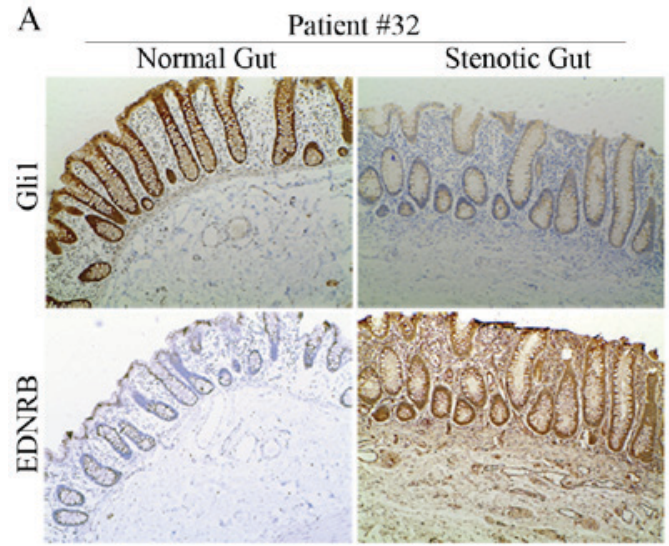

C

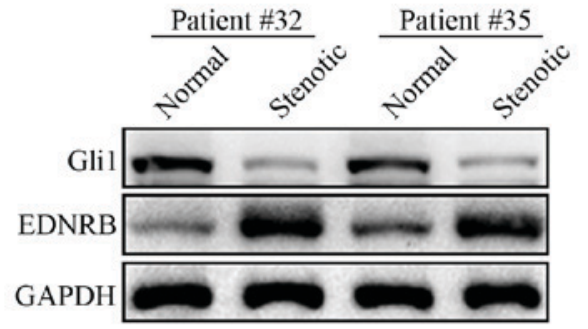

E

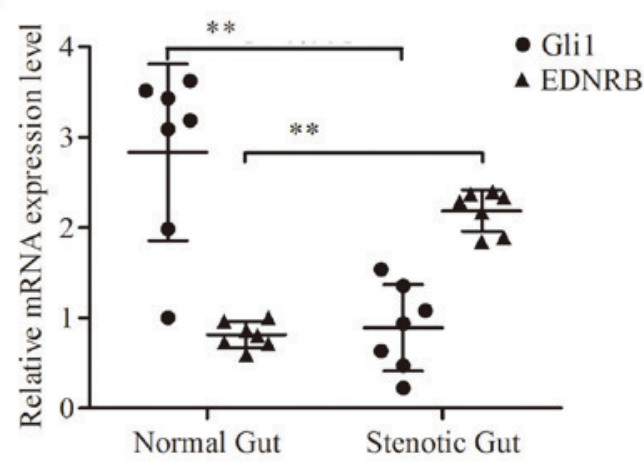

B

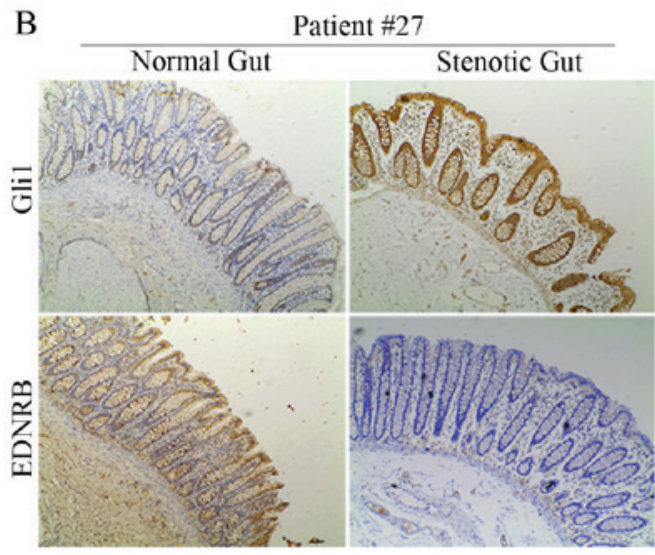

D

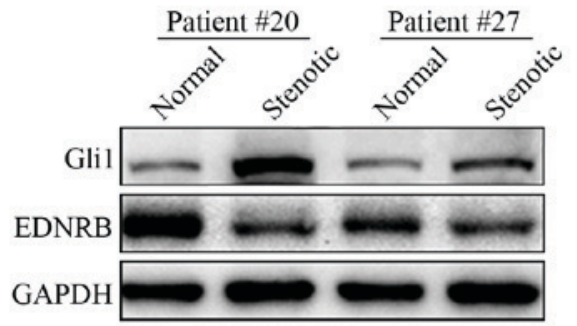

F

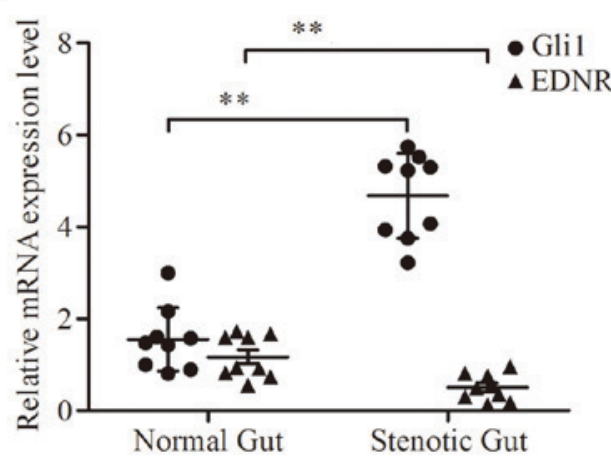

Figure 1. Gli1 and EDNRB expression in tissues from patients with HSCR. Upper and lower panels indicating Gli1 and EDNRB expression in (A) a low-level Gli1 expression sample from patient \#32 and (B) a high-level Gli1 expression sample from patient \#27 using immunohistochemistry. Magnification, x100; brown color, targeted proteins; blue color, nucleus; Representative western blot analysis of Gli1 and EDNRB expression in (C) low-level Gli1 samples from patient \#32 and \#35 and (D) high-level Gli1 specimens from patient \#20 and \#27, respectively. (E) Scatter plots for corresponding expression of Gli1 and EDNRB mRNA in (E) low-level Gli1 samples $(n=7)$ and $(F)$ high-level Gli1 samples $(n=9)$, respectively. Values are expressed as the mean \pm standard deviation. ${ }^{* *} \mathrm{P}<0.01$. Gli1, Gli family zinc finger 1; EDNRB, endothelin receptor type B; HSCR, Hirschsprung disease.

of HSCR was investigated by determining the expression of transcriptional factor Gli1 using immunohistochemical (IHC) analysis of tissue samples from 35 patients with HSCR. Gli1 expression was detected in the nucleus in normal and stenotic guts (Fig. 1A and B). The expression of Gill was decreased in $20 \%$ of the stenotic gut tissues, whereas it was increased in $28.57 \%$ of stenotic gut tissues compared with normal gut tissues. A previous study indicated that EDNRB is a major susceptibility gene in HSCR (21). Therefore, the expression of EDNRB was also determined in the same samples using IHC (Fig. 1A and B). Decreased expression of EDNRB was observed in $31.43 \%$ of stenotic gut tissues, whereas increased expression of EDNRB was identified in $20 \%$ of stenotic tissues compared with that in normal tissues. Aberrant expression of Gli1 and EDNRB suggests that Gli1 and EDNRB serve important roles in the pathogenesis of HSCR. Western blot analysis of lysates obtained from HSCR specimens confirmed the aberrant expression of Gli1 and
EDNRB in the stenotic gut (Fig. 1C and D), which is consistent with the IHC results.

Furthermore, RT-qPCR was performed to detect the expression of Gli1 and EDNRB mRNA in specimens. A total of 7 patients with decreased expression of Glil had high expression of EDNRB. The majority of patients with increased expression of Glil had low expression of EDNRB in stenotic guts (Fig. 1E and F). The results suggest that there is a negative association between the Hh and EDNRB signaling pathways.

Hh negatively regulates the expression of EDNRB in the $S K-N-S H$ cell line. The association between the $\mathrm{Hh}$ and EDNRB signaling pathways was determined using siRNA targeting Gli1 and a Gli1 expression plasmid. Knockdown of Gli1 markedly increased the expression of EDNRB (Fig. 2A), whereas upregulation of Glil markedly decreased EDNRB expression (Fig. 2B). 
A
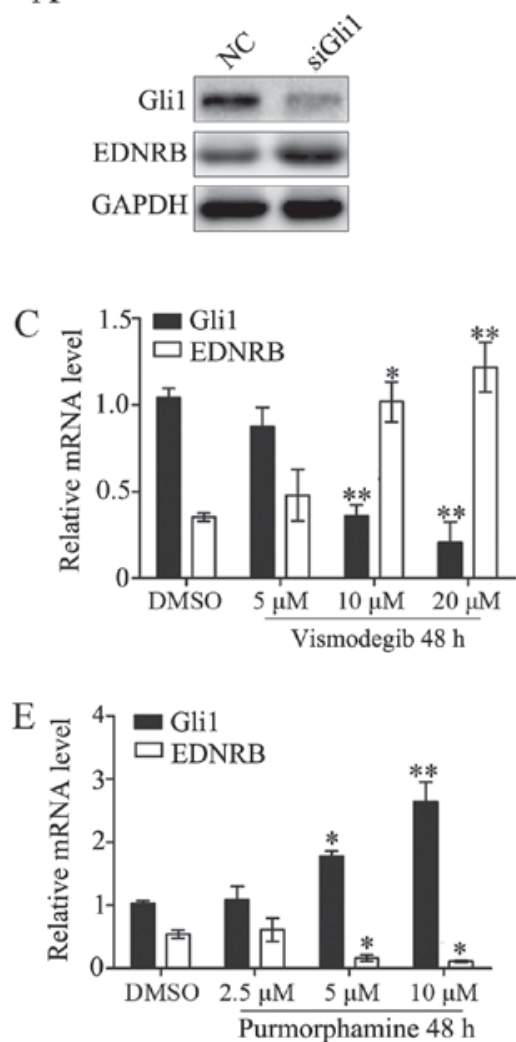

B

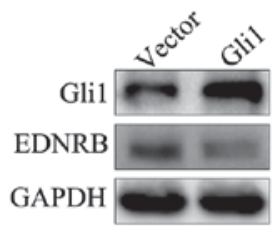

$\mathrm{D}$

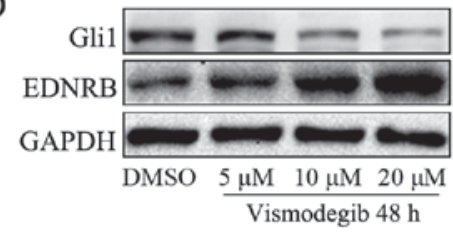

$\mathrm{F}$

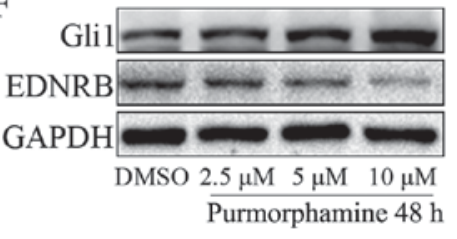

Figure 2. Hh negatively regulates EDNRB expression in the SK-N-SH cell line. (A) Protein expression of Gli1 and EDNRB in SK-N-SH cells following transfection with Gli1 siRNA and negative control siRNA using western blot analysis. (B) Protein expression of Gli1 and EDNRB in SK-N-SH cells stably transfected with Gli1 plasmid and control vector. (C) RT-qPCR and (D) western blot analysis were performed on cells treated with Vismodegib (5, 10 and $20 \mu \mathrm{M}$ ) or DMSO for $48 \mathrm{~h}$. (E) RT-qPCR and (F) western blot analysis were performed on cells treated with Purmorphamine (2.5, 5 and $10 \mu \mathrm{M})$ or DMSO for $48 \mathrm{~h}$. Values are expressed as the mean \pm standard deviation. " $\mathrm{P}<0.05 ;{ }^{* *} \mathrm{P}<0.01$ vs. DMSO groups. Hh, hedgehog; EDNRB, endothelin receptor type B; Gli1, Gli family zinc finger 1; siRNA, small interfering RNA; RT-qPCR, reverse transcription-quantitative polymerase chain reaction; DMSO, dimethyl sulfoxide.

Molecular regents were used to inhibit or activate $\mathrm{Hh}$ signaling. SK-N-SH cells were treated with Vismodegib $(5,10$ and $20 \mu \mathrm{M}$ for $48 \mathrm{~h}$ ) or Purmorphamine (2.5, 5 and 10 for $48 \mathrm{~h})$, with DMSO as a control. The expression of Gli1 and ENDRB mRNA and protein was detected using RT-qPCR and western blot analysis, respectively.

The expression of Gli1 was significantly decreased in the SK-N-SH cell line treated with 10 and $20 \mu \mathrm{M}$ Vismodegib for $48 \mathrm{~h}$, whereas the expression of EDNRB was significantly increased compared with cells treated with DMSO (Fig. 2C and D). By contrast, the activation of Hh signaling by 5 and $10 \mu \mathrm{M}$ Purmorphamine for $48 \mathrm{~h}$ significantly increased the expression of downstream molecular Glil, while the expression of EDNRB was significantly decreased compared with cells treated with DMSO (Fig. 2E and F). These results indicate that $\mathrm{Hh}$ negatively regulates EDNRB expression.

Effect of aberrant expression of Glil and EDNRB on ENC development in HSCR. Migration and differentiation are two important processes that serve a role in ENCC development. The genetic function of Hh/EDNRB in HSCR was investigated by analyzing the distribution and differentiation of ganglia using H\&E staining. There were 31 patients with HSCR with no enteric ganglia (Fig. 3) and 4 patients with HSCR and premature enteric ganglia in the stenotic intestine (Fig. 3). Decreased expression of Glil and increased expression of

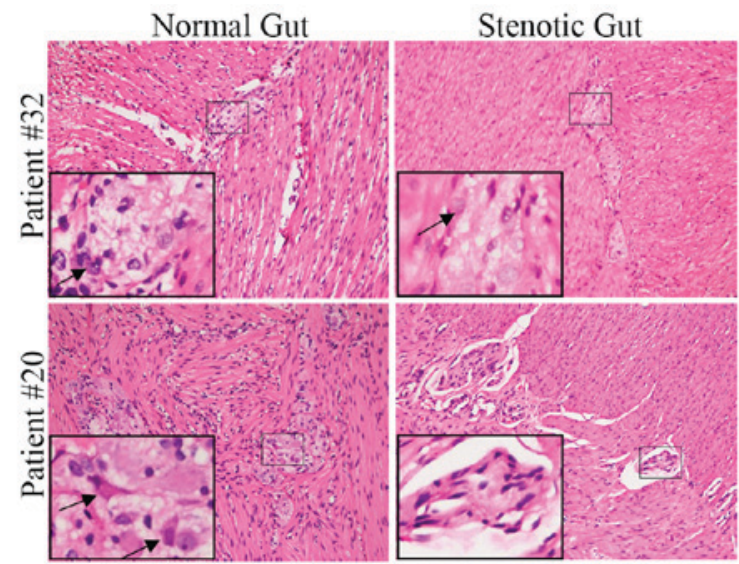

Figure 3. H\&E staining indicating ganglia in tissues from patients with HSCR. Upper panels indicate H\&E results for ganglia in a low-level Gli1 expression sample from patient \#32. Left image indicates mature ganglia in normal gut tissues and right image indicates premature ganglia in stenotic gut tissues. Lower panels indicate $\mathrm{H} \& \mathrm{E}$ results for ganglia in a high-level Gli1 expression specimen from patient \#20. Left image indicates mature ganglia in normal gut tissue and right image indicates a lack of ganglia in stenotic gut tissue. Magnification, x200. H\&E, hematoxylin and eosin; HSCR, Hirschsprung disease; Gli1, Gli family zinc finger 1.

EDNRB were exhibited in the patients with premature enteric ganglia. Taken together, these results suggest that decreased 
Glil expression may influence the differentiation of ENCCs via EDNRB.

\section{Discussion}

The ENS consists of innumerable ganglia cells, which aggregate by neurons and glial cells in gastrointestinal tract (4). During gut development, ENCC migration and differentiation are critical processes and coordination between them is important. HSCR is the primary disease caused by aberrant ENCC development. Disordered ENCC migration and neuronal/glial differentiation impede the complex physiological function of the gut (4). Surgery is the primary treatment strategy for HSCR; however, there are a range of long term prognoses in the majority of cases, including chronic constipation, fecal incontinence and Hirschsprung-associated enterocolitis (28).

Gut development, particularly the nervous system, is controlled by many genes (8). A single gene alteration has been associated with the pathogenesis a small proportion of HSCR patients. Integration of different signaling pathways is a critical process for development and the cumulative effects of alteration to several genetic pathways may increase the risk of HSCR. Furthermore, it has been demonstrated that the cross-talk between several pathways is important in the pathogenesis of HSCR (21). Increased expression of microRNA-218-1 correlates with increased levels of Slit homolog 2 protein and decreased levels of RET and zinc finger protein PLAG1, which leads to a loss of ganglion cells in the narrowed colon of mice (29). Ngan et al (30) also suggested that deletion of Patch reduces enteric progenitors and induces premature gliogenesis via activation of the Notch pathway. Hh and EDN3/EDNRB signaling pathways were regarded as the most important and the present study confirmed that Hh signaling regulates ENDRB expression in HSCR.

The genetic function of Hh signaling is a key aspect of ENCC migration and neuronal/glial differentiation (13). Protein patched homolog 1 is a negative regulator in the $\mathrm{Hh}$ signaling pathway whose deletion inhibits the neurogenesis process in the hindgut and is accompanied by spontaneous gliogenesis (14). Activation of Hh signaling via GLI1-3 mutations in HSCR has also been observed (31). In addition, the $\mathrm{Hh}$ signaling pathway negatively regulates other genes in many specific organs and diseases. For example, Hh signaling inhibits Wnt signaling via the canonical Wnt antagonist secreted frazzled related protein 1 in irradiated dying tumor cells (32). Suppression of Wnt/ $\beta$-catenin by $\mathrm{Hh}$ and protein-jagged 2 in the tongue epithelium may provide a novel strategy for disease treatment (33).

A previous study indicated that the methylation of EDNRB was decreased during HSCR (24). In the present stud, it was the expression of EDNRB in the aganglionic colon compared with the normal gut was assessed and upor downregulation was observed in a range of patients. The differential expression of EDNRB was the reverse of Gli1 and the negative association between Hh signaling and EDNRB remains unclear. Thus, SK-N-SH cells were used to clarify the mechanism underlying this effect. siRNA, plasmids and molecular regents Vismodegib and Purmorphamine were used to demonstrate that $\mathrm{Hh}$ negatively regulates EDNRB expression.

Taken together, the results of the present and previous studies suggest that alterations in gene expression contribute to the pathogenesis of HSCR. The distribution and differentiation of ganglia was also observed. The expression of Gli1 was decreased, whereas the expression of EDNRB was increased compared with the distal gut in 4 patients with HSCR and premature enteric ganglia in the narrow gut. No other patients exhibited enteric ganglia in the distal gut. This suggests that the absence of the Hh pathway in ENCCs may result in incomplete neuronal differentiation and constitutive activation of the Hh pathway may impede ENCC migration.

The results of the present study further highlight the role of genes in ENCC migration and differentiation in the pathogenesis of HSCR. The aberrant expression of Gli1 and ENDRB was demonstrated to be associated in HSCR. In addition, a novel molecular regulation mechanism was identified, in which Hh negatively regulated EDNRB expression in HSCR. Up- or downregulated Gli1 expression in HSCR was also observed in the present study. Glil downregulation inhibited neuronal differentiation, whereas Gli1 upregulation impeded ENCC migration. In conclusion, the results suggest that aberrant expression of Gli1 serves a role in HSCR by targeting EDNRB. This regulatory mechanism may lead to the development of novel therapeutic strategies for treating patients with HSCR.

\section{Acknowledgements}

The present study was supported by the National Natural Science Foundation of China (grant nos. 81600401 and 81572413), the Young Teacher Foundation of Independent Innovation Fund of Huazhong University of Science and Technology (grant no. 2015QN197), the Wuhan Scientific and Technological Application Foundation Project (grant no. 2015060101010044) and the Health and Family Plan Committee of China Research Fund of Public Welfare in Health Industry (grant no. 201402015).

\section{References}

1. Wetherill C and Sutcliffe J: Hirschsprung disease and anorectal malformation. Early Hum Dev 90: 927-932, 2014.

2. Pan ZW and Li JC: Advances in molecular genetics of Hirschsprung's disease. Anat Rec (Hoboken) 295: 1628-1638, 2012.

3. Skinner MA: Hirschsprung's disease. Curr Probl Surg 33: 389-460, 1996

4. Heanue TA and Pachnis V: Enteric nervous system development and Hirschsprung's disease: Advances in genetic and stem cell studies. Nat Rev Neurosci 8: 466-479, 2007.

5. Takawira C, D'Agostini S, Shenouda S, Persad R and Sergi C: Laboratory procedures update on Hirschsprung disease. J Pediatr Gastroenterol Nutr 60: 598-605, 2015

6. Schriemer D, Sribudiani Y, IJpma A, Natarajan D, MacKenzie KC, Metzger M, Binder E, Burns AJ, Thapar N, Hofstra RMW and Eggen BJL: Regulators of gene expression in Enteric Neural Crest Cells are putative Hirschsprung disease genes. Dev Biol 416: 255-265, 2016.

7. Bergeron KF, Silversides DW and Pilon N: The developmental genetics of Hirschsprung's disease. Clin Genet 83: 15-22, 2013.

8. Panza E, Knowles CH, Graziano C, Thapar N, Burns AJ, Seri M, Stanghellini V and De Giorgio R: Genetics of human enteric neuropathies. Prog Neurobiol 96: 176-189, 2012. 
9. Butler Tjaden NE and Trainor PA: The developmental etiology and pathogenesis of Hirschsprung disease. Transl Res 162: 1-15, 2013.

10. Mao J, Kim BM, Rajurkar M, Shivdasani RA and McMahon AP: Hedgehog signaling controls mesenchymal growth in the developing mammalian digestive tract. Development 137: 1721-1729, 2010.

11. Hui CC and Angers S: Gli proteins in development and disease. Annu Rev Cell Dev Biol 27: 513-537, 2011.

12. Huang H, Cotton JL, Wang Y, Rajurkar M, Zhu LJ, Lewis BC and Mao J: Specific requirement of Gli transcription factors in Hedgehog-mediated intestinal development. J Biol Chem 288 17589-17596, 2013.

13. Charrier B and Pilon N: Toward a better understanding of enteric gliogenesis. Neurogenesis (Austin) 4: e1293958, 2017.

14. Nagy N, Barad C, Graham HK, Hotta R, Cheng LS, Fejszak N and Goldstein AM: Sonic hedgehog controls enteric nervous system development by patterning the extracellular matrix. Development 143: 264-275, 2016.

15. Ramalho-Santos M, Melton DA and McMahon AP: Hedgehog signals regulate multiple aspects of gastrointestinal development. Development 127: 2763-2772, 2000.

16. Fu M, Lui VC, Sham MH, Pachnis V and Tam PK: Sonic hedgehog regulates the proliferation, differentiation, and migration of enteric neural crest cells in gut. J Cell Biol 166: 673-684, 2004.

17. Liu JA, Lai FP, Gui HS, Sham MH, Tam PK, Garcia-Barcelo MM, Hui CC and Ngan ES: Identification of GLI mutations in patients with hirschsprung disease that disrupt enteric nervous system development in mice. Gastroenterology 149: 1837-1848.e5, 2015

18. Baynash AG, Hosoda K, Giaid A, Richardson JA, Emoto N, Hammer RE and Yanagisawa M: Interaction of endothelin-3 with endothelin-B receptor is essential for development of epidermal melanocytes and enteric neurons. Cell 79: 1277-1285, 1994

19. Bondurand N, Natarajan D, Barlow A, Thapar N and Pachnis V: Maintenance of mammalian enteric nervous system progenitors by SOX10 and endothelin 3 signalling. Development 133 2075-2086, 2006.

20. Kenny SE, Hofstra RM, Buys CH, Vaillant CR, Lloyd DA and Edgar DH: Reduced endothelin-3 expression in sporadic Hirschsprung disease. Br J Surg 87: 580-585, 2000.

21. Sergi CM, Caluseriu O, McColl $\mathrm{H}$ and Eisenstat DD: Hirschsprung's disease: Clinical dysmorphology, genes, micro-RNAs, and future perspectives. Pediatr Res 81: 177-191, 2017.

22. Druckenbrod NR and Epstein ML: Age-dependent changes in the gut environment restrict the invasion of the hindgut by enteric neural progenitors. Development 136: 3195-3203, 2009.
23. Chen B, Ouyang HL, Wang WH, Yin YH, Yan LN, Yang B and Xue ZF: Hirschsprung disease is associated with an L286P mutation in the fifth transmembrane domain of the endothelin-B receptor in the N-ethyl-N-nitrosourea-induced mutant line. Exp Anim 65: 245-251, 2016

24. Tang W, Li B, Tang J, Liu K, Qin J, Wu W, Geng Q, Zhang J, Chen H, Xu X and Xia Y: Methylation analysis of EDNRB in human colon tissues of Hirschsprung's disease. Pediatr Surg Int 29: 683-688, 2013.

25. Chaturvedi NK, McGuire TR, Coulter DW, Shukla A, McIntyre EM, Sharp JG and Joshi SS: Improved therapy for neuroblastoma using a combination approach: Superior efficacy with vismodegib and topotecan. Oncotarget 7: 15215-15229, 2016.

26. Livak KJ and Schmittgen TD: Analysis of relative gene expression data using real-time quantitative PCR and the 2(-Delta Delta $\mathrm{C}(\mathrm{T})$ ) method. Methods 25: 402-408, 2001

27. Löf Granström A, Svenningsson A, Hagel E, Oddsberg J, Nordenskjöld A and Wester T: Maternal risk factors and perinatal characteristics for hirschsprung disease. Pediatrics 138: pii: e20154608, 2016

28. Menezes M, Corbally M and Puri P: Long-term results of bowel function after treatment for Hirschsprung's disease: A 29-year review. Pediatr Surg Int 22: 987-990, 2006.

29. Tang W, Tang J, He J, Zhou Z, Qin Y, Qin J, Li B, Xu X, Geng Q, Jiang W, et al: SLIT2/ROBO1-miR-218-1-RET/PLAG1: A new disease pathway involved in Hirschsprung's disease. J Cell Mol Med 19: 1197-1207, 2015

30. Ngan ES, Garcia-Barceló MM, Yip BH, Poon HC, Lau ST, Kwok CK, Sat E, Sham MH, Wong KK, Wainwright BJ, et al: Hedgehog/Notch-induced premature gliogenesis represents a new disease mechanism for Hirschsprung disease in mice and humans. J Clin Invest 121: 3467-3478, 2011.

31. Young HM, Stamp LA and Hofstra RM: Hirschsprung disease and activation of hedgehog signaling via GLI1-3 mutations. Gastroenterology 149: 1672-1675, 2015.

32. Ma J, Cheng J, Gong Y, Tian L and Huang Q: Downregulation of Wnt signaling by sonic hedgehog activation promotes repopulation of human tumor cell lines. Dis Model Mech 8: 385-391, 2015.

33. Schneider FT, Schänzer A, Czupalla CJ, Thom S, Engels K, Schmidt MH, Plate KH and Liebner S: Sonic hedgehog acts as a negative regulator of $\{$ beta\}-catenin signaling in the adult tongue epithelium. Am J Pathol 177: 404-414, 2010.

This work is licensed under a Creative Commons

Attribution-NonCommercial-NoDerivatives 4.0 International (CC BY-NC-ND 4.0) License. 